\title{
Large-Scale Automated Analysis of Location Patterns in Randomly Tagged $3 T 3$ Cells
}

\author{
Elvira García Osuna, ${ }^{1,2}$ Juchang Hua, ${ }^{1,3,4}$ Nicholas W. Bateman, ${ }^{3}$ Ting Zhao, ${ }^{1,2}$ Peter B. Berget, ${ }^{3}$ \\ and Robert F. Murphy ${ }^{1,2,3,4}$

\begin{abstract}
${ }^{1}$ Center for Bioimage Informatics, HHC119, Carnegie Mellon University, 5000 Forbes Ave., Pittsburgh, PA 15213, USA; Carnegie Mellon University, Pittsburgh, PA, USA; and ${ }^{4}$ Department of Machine Learning, Carnegie Mellon University, Pittsburgh, PA, USA
\end{abstract} \\ ${ }^{2}$ Department of Biomedical Engineering, Carnegie Mellon University, Pittsburgh, PA, USA; ${ }^{3}$ Department of Biological Sciences,
}

(Received 22 September 2006; accepted 4 January 2007; published online 7 February 2007)

\begin{abstract}
Location proteomics is concerned with the systematic analysis of the subcellular location of proteins. In order to perform high-resolution, high-throughput analysis of all protein location patterns, automated methods are needed. Here we describe the use of such methods on a large collection of images obtained by automated microscopy to perform high-throughput analysis of endogenous proteins randomly-tagged with a fluorescent protein in NIH 3 T3 cells. Cluster analysis was performed to identify the statistically significant location patterns in these images. This allowed us to assign a location pattern to each tagged protein without specifying what patterns are possible. To choose the best feature set for this clustering, we have used a novel method that determines which features do not artificially discriminate between control wells on different plates and uses Stepwise Discriminant Analysis (SDA) to determine which features do discriminate as much as possible among the randomly-tagged wells. Combining this feature set with consensus clustering methods resulted in 35 clusters among the first 188 clones we obtained. This approach represents a powerful automated solution to the problem of identifying subcellular locations on a proteome-wide basis for many different cell types.
\end{abstract}

Keywords-Protein subcellular location, Subcellular location trees, Subcellular location features, CD-tagging, Fluorescence microscopy, Cluster analysis, Location proteomics.

\section{INTRODUCTION}

Current work in proteomics includes systematic analysis of protein structure, expression levels, and interactions. These projects will provide critical data for understanding and modeling cell and tissue behavior. Knowledge of the subcellular location of

Address correspondence to Robert F. Murphy, Center for Bioimage Informatics, HHC119, Carnegie Mellon University, 5000 Forbes Ave., Pittsburgh, PA, 15213, USA. Electronic mail: murphy@ cmu.edu each protein is equally important to this task. However, this area has received far less attention.

There are two major ways of analyzing protein subcellular location: prediction and determination. A number of systems for predicting protein localization from sequence have been described. ${ }^{5,8,14,17,18}$ The limitation of these systems is that they can only assign new proteins to the location categories with which they have been trained. This means that proteins with previously unseen location patterns cannot be properly categorized. In addition, since they have been trained to recognize only low-resolution classes, they are typically able to predict the organelle to which a protein will be localized, but not the specific area of the organelle. Due to lack of training data, they are also unable to predict differential localization of proteins in different cell types or under different conditions.

\section{Determination of Protein Location}

Due to the limitations of prediction, there is a need for projects that will collect data on subcellular location for entire proteomes under a variety of conditions. These projects determine protein location rather than predict it. Although these projects are useful in their own right, they also serve as a way to expand the capabilities of prediction systems by providing training examples for higher-resolution and complex patterns.

Fluorescence microscopy has been widely used for determining protein subcellular location, and visual examination has been the primary means of analyzing the resulting images. Some large-scale projects have used fluorescence microscopy to screen hundreds to thousands or proteins for particular patterns or to assign proteins to major location classes. ${ }^{11,13,20,22}$ A particular ambitious and valuable project has been the 
tagging of all predicted protein coding regions in the yeast Saccharomyces cerevisiae. ${ }^{11}$

Visual examination of images is not only inefficient for high-throughput projects, but it is also subjective and irreproducible. Fortunately, automated methods of analyzing protein location have been described by our group ${ }^{1-3,10}$ and more recently by others. ${ }^{6,7,21}$ These methods have been shown not only to perform as well as visual examination for distinguishing major subcellular patterns, but also to be able to discriminate patterns that a human observer cannot. ${ }^{16}$

There is not only a need for automated analysis of images, but large-scale projects also require highthroughput methods for acquiring images. Automated fluorescence microscopes originally developed for drug screening can meet this need. ${ }^{19,23}$ These microscopes use multi-well plates, contain autofocus capabilities and are capable of multi-color imaging as well as $3 \mathrm{D}$ time-series imaging.

\section{CD-Tagging of NIH 3 T3 Cells}

In order to perform systematic analysis of protein location by fluorescence microscopy, a high-throughput means of tagging all (or most) proteins is also needed. One such method is CD-tagging. ${ }^{12}$ This method inserts a guest exon into genomic DNA. The insert consists of an enhanced green fluorescent protein (EGFP) coding sequence flanked by splicing signals. Therefore, when the protein with the guest exon insertion is expressed, it contains an internal fluorescent tag. Previous studies have shown that $\mathrm{CD}$-tagging has minimal impact on protein folding, function and localization. ${ }^{13}$ Here, we combine CD-tagging, automated microscopy and automated analysis to identify statistically distinguishable location patterns NIH 3T3 cells. We present the combination of high-throughput methods from tagging to analysis as well as fully automated methods of imaging and analysis.

\section{METHODS}

\section{Production and Isolation of CD-Tagged NIH 3 T3 Cells}

The procedure described previously ${ }^{13}$ was followed, with some minor alterations. A CD-tagging cassette containing the EGFP coding sequence was packaged into retrovirus using Phoenix-GP cells. Phoenix-GP cells were seeded at a rate of $1.3 \times 10^{6}$ cells per $75 \mathrm{~cm}^{2}$ flask in complete Phoenix media (Dulbecco's Modified Eagle's Medium (DMEM) containing 10\% fetal bovine serum). The Phoenix-GP cells were transfected the next day with $9 \mu \mathrm{g}$ Stealth plasmid and $1 \mu \mathrm{g}$ VSV-G plasmid per flask using Mirus Trans-IT-LT-1 lipofection reagent as per manufacturer's protocol. Briefly, $15 \mu \mathrm{l}$ Trans-IT-LT- 1 was added to $500 \mu \mathrm{l}$ serum-free media and incubated for $5 \mathrm{~min}$ at room temperature. The DNA was then added to this mixture, which was then incubated for an additional $20 \mathrm{~min}$ at room temperature. The resulting DNA complexes were then added to the Phoenix-GP cells in $10 \mathrm{ml}$ fresh complete media and the cells were incubated for $24 \mathrm{~h}$ at $37^{\circ} \mathrm{C}$ and $5 \%$ $\mathrm{CO}_{2}$. After $24 \mathrm{~h}$, the media was replaced with $10 \mathrm{ml}$ fresh media and the flasks were incubated at $32^{\circ} \mathrm{C}$ and $5 \% \mathrm{CO}_{2}$ for $48 \mathrm{~h}$. The resulting viral supernatant was flash frozen in $1 \mathrm{ml}$ aliquots in liquid nitrogen and stored at $-80^{\circ} \mathrm{C}$. Viral supernatants were created using three different versions of the Stealth plasmid, P19, P20 and P21, which encode EGFP appropriately for class 0 , class 1 and class 2 introns, respectively. A different virus was used each week so that introns of all types could be sampled.

For infection, NIH $3 \mathrm{~T} 3$ cells were plated at $2 \times 10^{5}$ cells per well of a 6-well plate containing complete media (DMEM containing 10\% fetal calf serum, $100 \mathrm{U} / \mathrm{ml}$ penicillin, and $100 \mu \mathrm{g} / \mathrm{ml}$ streptomycin). Six hours later, the media was aspirated and viral supernatant was added with $6 \mu \mathrm{g} / \mathrm{ml}$ polybrene (to neutralize the charge on the cell surface so that viral particles will not be repelled) and incubated for $24 \mathrm{~h}$ at $37^{\circ} \mathrm{C}$ and $5 \% \mathrm{CO}_{2}$.

The cells were then trypsinized, expanded into a $10 \mathrm{~cm}$ dish and incubated for $48 \mathrm{~h}$. EGFP-expressing cells were sorted using a FACS Vantage SE using a threshold set to include only $0.1 \%$ of untagged, control cells. Positive, singlet cells were sorted into black polystyrene, glass-bottomed 96-well plates (Whatman) containing $200 \mu \mathrm{l}$ of complete medium (Dulbecco's modified Eagles medium, 10\% fetal calf serum, $100 \mathrm{U} /$ $\mathrm{ml}$ penicillin and $100 \mu \mathrm{g} / \mathrm{ml}$ streptomycin). Plates were incubated for 8 days before adding $1 \times 10^{4}$ untagged and positive control cells to one well each in each row (cells expressing tagged Procollagen Type I $\alpha 2$ were used as the positive control).

On days $11-15$, the media was aspirated and the DNA-binding vital dye Hoechst 33342 was added at a concentration of $0.5 \mu \mathrm{g} / \mathrm{ml}$ in OptiMEM (Invitrogen Corporation, Carlsbad, CA, USA). Plates were then incubated for $45 \mathrm{~min}$ at $37^{\circ} \mathrm{C}$ and $5 \% \mathrm{CO}_{2}$ before imaging.

\section{Automated Fluorescence Imaging}

Two color images (Hoechst 33342 and EGFP) were acquired using an automated fluorescence microscope (Beckman Coulter IC-100). Images were acquired with a $40 \times 0.9$ NA objective and a Hamamatsu Orca-ERG camera at a fixed camera gain and exposure time. Twenty-five fields were imaged for each well using 
autofocus on the Hoechst channel. Images of empty wells were discarded. The remaining images of EGFPpositive cells were used for analysis.

\section{Feature Calculation and Selection}

The most common approach to describing subcellular pattern is to use features calculated on single cell images. This requires segmenting each image into single cell regions, a task that can be quite error prone. For the large number of images in this study, we therefore used a new set of our Subcellular Location Features that are not sensitive to the number of cells in an image. The starting point for this set was SLF21, which has previously been shown to provide good performance for classifying subcellular patterns without cell segmentation. ${ }^{9}$ It includes 3 morphological features, 5 edge features and 13 Haralick texture features. We augmented this set by calculating the 13 Haralick texture features after downsampling the protein image from two to six fold and adding a new feature which measures the percentage of pixels that are above threshold in the protein (EGFP) image which are also above threshold in the DNA (Hoechst) image. (Thresholding is performed as described previously. ${ }^{9}$ ) These additional 66 features gave us a total of 87 features to describe each image. We define this set as SLF25.

To assess the sensitivity of a given feature to undesirable well-to-well and plate-to-plate variation, $t$-tests were performed for all pairs of images (fields) of positive control cells. Average $p$-values were calculated for all pairwise tests for a given feature, and various thresholds on this average were used for feature elimination.

Step discriminant analysis (SDA) was then done for the remaining features on the entire image dataset to select those with good discriminating power: the features that can differentiate the patterns.

\section{Clustering of Protein Patterns}

A three-step process was used to cluster the wells that contained tagged proteins. First, $k$-means clustering with a $z$-scored Euclidean distance function was performed on the image varying $k$ from 1 to 100 . Akaike Information Criterion (AIC) was then calculated to select an optimal $k$ and corresponding clustering of the images. Second, each well was allocated to that cluster which contains a plurality of the images in the well and only the images in this cluster were kept for further analysis. If, however, the number of images assigned to the plurality cluster was less than $1 / 3$ of the total number of images for a given well, that well was considered not to have a unique pattern and it was removed from the analysis.

Lastly, a consensus tree algorithm ${ }^{4}$ was applied to the remaining images. In this algorithm, a hierarchical cluster tree (dendrogram), was generated from a random half of images of each well. This was repeated 200 times and a consensus tree was generated in which only the branches of the trees that were present in at least half of the trees were kept.

Visual inspection was also used to cluster the tagged wells. During this process, descriptive terms were assigned to each well by one of the authors (EGO) after carefully examining the representative images of each well (representative images were chosen as described previously ${ }^{15}$ ). Whatever terms that were felt to accurately describe the protein pattern were used, and for the consistency, the same terms were used for the same patterns. Wells were then grouped into those that shared a unique combination of the descriptive terms.

In order to measure the agreement of different clustering results, we calculated Cohen's $\kappa$ statistics on each pair of clustering results $\mathrm{A}$ and $\mathrm{B}$ :

$k(A, B)=\frac{\text { Observed agreement }- \text { expected agreement }}{1-\text { expected agreement }}$

where expected agreement is that expected for two random samplings from the same clustering. ${ }^{4}$

\section{Software and Data Availability}

All data and Matlab code used in this paper are available at http://murphylab.web.cmu.edu/data and http://murphylab.web.cmu.edu/software, respectively.

\section{RESULTS}

We have previously demonstrated the feasibility of automated clustering of randomly tagged proteins by their location pattern using high-resolution images obtained with a spinning disk confocal microscope. This required major efforts in three areas: time and culture expense for isolating, expanding and maintaining individual clones, large reagent costs for identifying the tagged gene by RT-PCR and sequencing, and extensive time for individually plating and carrying out 3D imaging for each clone. The results provided information about the location of each protein but also about the number and type of patterns that were observed. Given the expense of this approach, we sought to evaluate a much less expensive alternative for just determining the set of possible patterns: sorting 

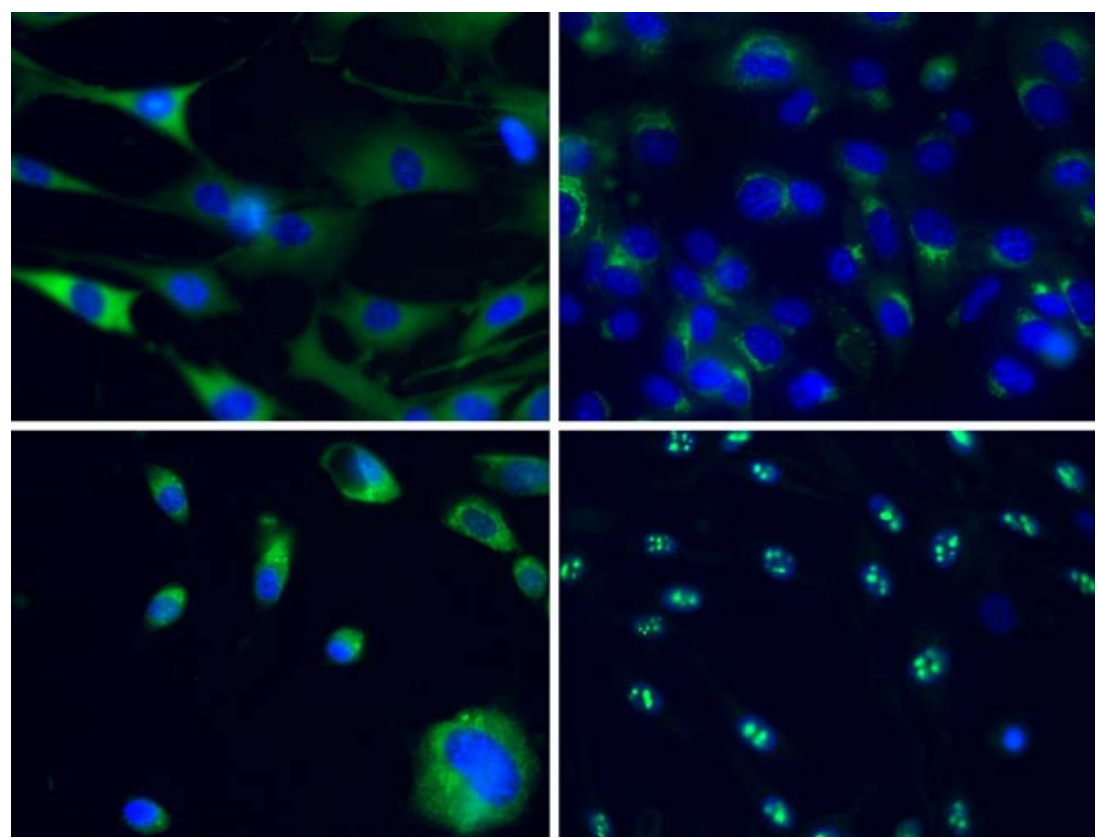

FIGURE 1. Example images from the dataset acquired in this study. The clones varied in protein expression level, and therefore each panel was fully contrast-stretched to facilitate visualization (hence the background appears different in each panel).

individual tagged cells directly into 96-well plates and imaging them without identifying the tagged gene. To test the feasibility of this approach, we generated and imaged ten plates per week for four weeks. After eliminating edge rows and columns (which could not be imaged due to interference by the plate skirt with the $40 \times$ objective) and the negative and positive control wells (three each per plate), we obtained images for 54 randomly tagged wells per plate or a total of 2160 wells. Of these, 222 contained EGFP-positive cells. Examples of these images can be seen in Fig. 1. After removing those images which were overcrowded or those for which valid features could not be calculated due to low fluorescence signal, a total of 174 wells with at least 10 images remained. These were used in clustering analysis.

An important issue for any image clustering approach is the nature of the features to be used. Given that the images we wished to cluster were collected on different days over many weeks (albeit under nominally the same conditions each day), one concern in this respect is that features that are sensitive to day-today variations might result in clustering proteins by day of acquisition (or position within a plate) rather than by protein pattern. The presence of the positive controls wells in each plate allowed us to design a strategy to minimize this concern. We sought to select features that can tell the difference between the different randomly tagged proteins but not be sensitive to the variance among the positive controls from plate to plate. As described in the Methods, we did extensive $t$-tests on each feature for each pair of images from control wells to eliminate features that were significantly different between the controls. We used three thresholds $(0,0.1$ and 0.2$)$ on the average $p$-values to eliminate plate dependent features. The remaining features were then subjected to stepwise discriminant analysis (SDA) to eliminate features that did not provide any discriminating power between the randomly tagged wells. A total of 76, 64 and 42 features were retained for thresholds of $0,0.1$ and 0.2 , respectively.

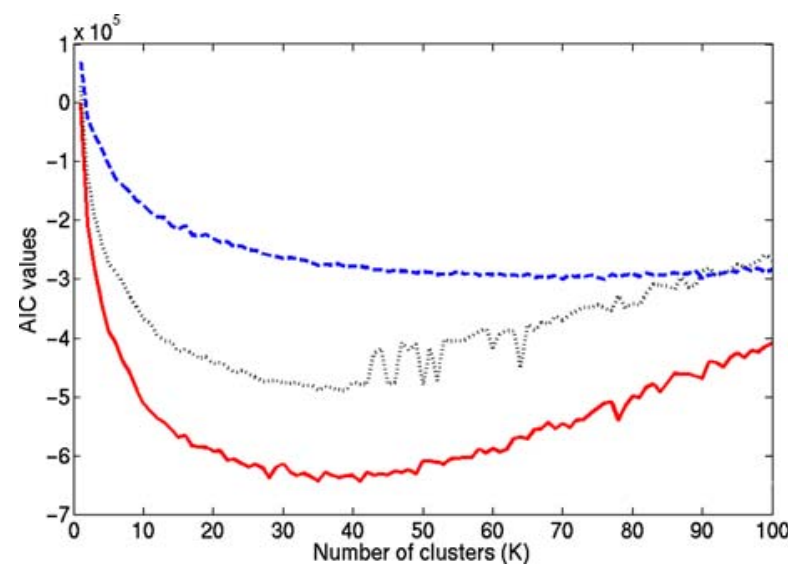

FIGURE 2. Determination of the optimal number of clusters using AIC. Three p-value thresholds were used (solid: 0, dashed: 0.1, dotted: 0.2 ) to select a set of features and then $k$-means clustering was performed for various values of $k$. AIC was calculated to measure the goodness of each clustering. The optimal values of $k$ are 41,35 and 70 , respectively. 

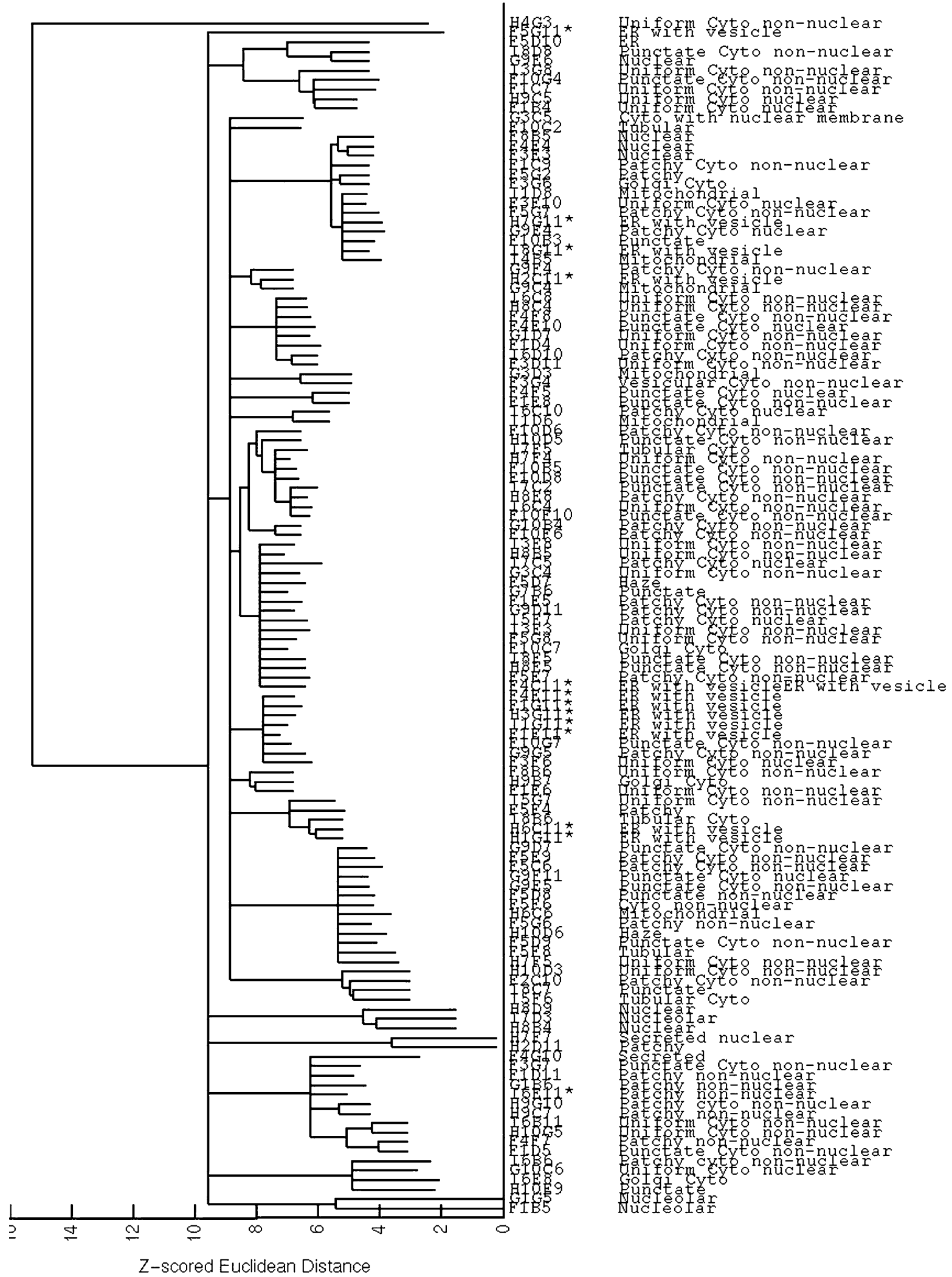

\section{Z-scored Euclidean Distance}

FIGURE 3. A consensus subcellular location tree built from 126 wells of the randomly tagged 3 T3 image dataset. A threshold of 0.1 was used on the average $p$-value of the statistic tests on control wells to select features. The first column of labels shows the well name and (positive control wells are marked with an asterisk). The second column of labels shows the locations assigned by visual inspection. In this tree, the sum of the lengths of the branches connecting two clones is proportional to the distance between them in feature space. 
Using these features, we then performed k-means clustering on all images for the 174 clones (plus 14 positive control wells) for various values of $k$ (the number of clusters). The goodness of these different clustering runs was evaluated using the Akaike Information Criterion (AIC), which balances tightness of the clusters against the number of clusters. These AIC values are plotted as a function of $k$ in Fig. 2. The results indicate that the optimal numbers of clusters are 41,35 and 70 for the feature sets selected using a $p$-value threshold of $0,0.1$ and 0.2 .

Consensus trees were then built for each feature set. These can be viewed through a web interface at http:// murphylab.web.cmu.edu/services/PSLID that permits display of representative images for each well. The consensus tree built with a $p$-value threshold of 0.1 is shown in Fig. 3.

Different feature sets led to different clustering results. In order to measure how much they agree with each other, the Cohen $\kappa$ statistics was calculated for each pair of clustering results. Since different sets of clones were retained in each final clustering, only the common clones in both clustering results were considered in each calculation. Additionally, labels of subcellular location patterns were assigned to each well by visual inspection (shown in Fig. 3), and a clustering was generated by grouping wells with the same labels. The Cohen $\kappa$ statistics was also calculated between visual inspection and all three automated clustering results. The results are shown in Table 1. The agreements between visual inspection and $k$-means clustering results are obviously lower than those between different $k$-means clustering results. This indicates the consistency of automated methods of cluster analysis.

\section{DISCUSSION}

We have described a high-throughput method of analyzing randomly tagged NIH $3 \mathrm{~T} 3$ cells. This method is automated and results in clusters of protein patterns that have similar distributions. This method allows us to analyze images without any previous

TABLE 1. Comparison of clustering results. Cohen's $\kappa$ statistic was calculated to measure the degree of agreement between each pair of clustering results.

\begin{tabular}{|c|c|c|c|c|}
\hline & & \multicolumn{3}{|c|}{$k$-Means/AIC with $p$-value threshold } \\
\hline & & 0 & 0.1 & 0.2 \\
\hline \multicolumn{2}{|l|}{ Visual inspection } & $0.13(0.01)$ & $0.06(0.01)$ & $0.18(0.03)$ \\
\hline k-Means/AIC & 0 & & $0.36(0.02)$ & $0.75(0.04)$ \\
\hline & 0.1 & & & $0.49(0.04)$ \\
\hline
\end{tabular}

Higher values indicate better agreement. The numbers in the parentheses are the standard deviation of the statistics. knowledge of the protein subcellular location. The work is distinguished from our prior work in that we describe a higher throughput pipeline for infecting, sorting and imaging tagged lines, the use of a internal control and a modified feature selection procedure to minimize the effects of variability during the imaging process, and the use of a new set of field level features that do not require segmentation into single cells.

It should be noted that in the work described here only proteins for which a consistent location pattern could be found were analyzed. Future work will extend the analysis to identify proteins with variable patterns, such as those that show cell cycle dependence. The data collected in this study are being made publicly available to facilitate development of methods for this type of analysis.

The current results show that many, but not all, of the positive controls were clustered together. This suggests that additional effort is needed in the future to ensure consistency between different runs. Incorporating a larger number of positive controls that represent additional major subcellular locations would therefore appear useful. We are adopting this approach in our ongoing experiments to expand our database to include thousands of tagged proteins. Our goal is then to use cluster analysis as described here to determine the number and types of subcellular location families that are present in NIH 3T3 cells. Once the set of possible patterns is known, the methods described here can be used to screen for clones with particular patterns so that the tagged gene can be sequenced. This will be useful for identifying novel patterns and proteins that display them as well as providing new data for training location prediction methods.

\section{ACKNOWLEDGMENTS}

We would like to thank Dr. Jonathan Jarvik for helpful discussions and Yehuda Creeger for technical assistance. This work was supported by Commonwealth of Pennsylvania Tobacco Settlement Fund grant 017393, NIH grant GM068845-01, and NSF grant EF-0331657.

\section{REFERENCES}

\footnotetext{
${ }^{1}$ Boland, M. V., M. K. Markey, and R. F. Murphy. Classification of protein localization patterns obtained via fluorescence light microscopy. In Proc of 19th Annual International Conference of the IEEE Engineering in Medicine and Biology Society, pp. 594-597, 1997.

${ }^{2}$ Boland, M. V., M. K. Markey, and R. F. Murphy. Automated recognition of patterns characteristic of
} 
subcellular structures in fluorescence microscopy images. Cytometry 33:366-375, 1998.

${ }^{3}$ Boland, M. V. and R. F. Murphy. A neural network classifier capable of recognizing the patterns of all major subcellular structures in fluorescence microscope images of hela cells. Bioinformatics 17:1213-1223, 2001.

${ }^{4}$ Chen, X. and R. F. Murphy. Objective clustering of proteins based on subcellular location patterns. J. Biomed. Biotechnol. 2005:87-95, 2005.

${ }^{5}$ Chou, K. C. and Y. D. Cai. Prediction and classification of protein subcellular location-sequence-order effect and pseudo amino acid composition. J. Cell Biochem. 90:1250 1260, 2003.

${ }^{6}$ Conrad, C., H. Erfle, P. Warnat, N. Daigle, T. Lorch J. Ellenberg, R. Pepperkok, and R. Eils. Automatic identification of subcellular phenotypes on human cell arrays. Genome Res. 14:1130-1136, 2004.

${ }^{7}$ Danckaert, A., E. Gonzalez-Couto, L. Bollondi N. Thompson, and B. Hayes. Automated recognition of intracellular organelles in confocal microscope images. Traffic 3:66-73, 2002.

${ }^{8}$ Guda, C., E. Fahy, and S. Subramaniam. Mitopred: A genome-scale method for prediction of nucleus-encoded mitochondrial proteins. Bioinformatics 20:Jul 22, 2004.

${ }^{9}$ Huang, K., and R. F. Murphy. Automated classification of subcellular patterns in multicell images without segmentation into single cells. In Proc of 2004 IEEE International Symposium on Biomedical Imaging (ISBI-2004), pp. 1139$1142,2004$.

${ }^{10}$ Huang, K. and R. F. Murphy. Boosting accuracy of automated classification of fluorescence microscope images for location proteomics. BMC Bioinformatics 5:78.

${ }^{11}$ Huh, W.- K., J. V. Falvo, L. C. Gerke, A. S. Carroll, R. W. Howson, J. S. Welssman, and E. K. O'Shea. Global analysis of protein localization in budding yeast. Nature 425:686-691, 2003.

${ }^{12}$ Jarvik, J. W., S. A. Adler, C. A. Telmer, V. Subramaniam, and A. Lopez. Cd-tagging: A new approach to gene and protein discovery and analysis. BioTechniques 20:896-904, 1996.

${ }^{13}$ Jarvik, J. W., G. W. Fisher, C. Shi, L. Hennen, C. Hauser, S. Adler, and P. B. Berget. In vivo functional proteomics: Mammalian genome annotation using cd-tagging. BioTechniques 33:852-867, 2002.
${ }^{14}$ Lu, Z., D. Szafron, R. Greiner, P. Lu, D. S. Wishart B. Poulin, J. Anvik, C. Macdonell, and R. Eisner. Predicting subcellular localization of proteins using machinelearned classifiers. Bioinformatics 20:547-556, 2004.

${ }^{15}$ Markey, M. K., M. V. Boland, and R. F. Murphy. Towards objective selection of representative microscope images. Biophys. J. 76:2230-2237, 1999.

${ }^{16}$ Murphy, R. F., M. Velliste, and G. Porreca. Robust numerical features for description and classification of subcellular location patterns in fluorescence microscope images. J. VLSI Sig. Proc. 35:311-321, 2003.

${ }^{17}$ Nakai, K.. Protein sorting signals and prediction of subcellular localization. Adv. Protein Chem. 54:277-344, 2000.

${ }^{18}$ Park, K. J. and M. Kanehisa. Prediction of protein subcellular locations by support vector machines using compositions of amino acids and amino acid pairs. Bioinformatics 19:1656-1663, 2003.

${ }^{19}$ Price, J. H., A. Goodacre, K. Hahn, L. Hodgson, E. A. Hunter, S. Krajewski, R. F. Murphy, A. Rabinovich, J. C. Reed, and S. Heynen. Advances in molecular labeling, high throughput imaging and machine intelligence portend powerful functional cellular biochemistry tools. J. Cell. Biochem. Supp. 39:194-210, 2002.

${ }^{20}$ Rolls, M. M., P. A. Stein, S. S. Taylor, E. Ha, F. McKeon, and T. A. Rapoport. A visual screen of a gfp-fusion library identifies a new type of nuclear envelope membrane protein. J. Cell Biol. 146:29-44, 1999.

${ }^{21}$ Sigal, A., R. Milo, A. Cohen, N. Geva-Zatorsky, Y. Klein, I. Alaluf, N. Swerdlin, N. Perzov, T. Danon, Y. Liron, T. Raveh, A. E. Carpenter, G. Lahav, and U. Alon. Dynamic proteomics in individual human cells uncovers widespread cell-cycle dependence of nuclear proteins. Nat. Methods 3:525-531, 2006.

${ }^{22}$ Simpson, J. C., R. Wellenreuther, A. Poustka, R. Pepperkok, and S. Wiemann. Systematic subcellular localization of novel proteins identified by large-scale cDNA sequencing. EMBO Rep. 1:287-292, 2000.

${ }^{23}$ Taylor, D. L., E. S. Woo, and K. A. Giuliano. Real-time molecular and cellular analysis: The new frontier of drug discovery. Curr. Opin. Biotechnol. 12:75-81, 2001. 